\title{
Implement of Smart Health Care Monitoring System using Mobile IoT and Cloud Computing Technologies
}

\author{
K. V. Daya Sagar
}

\begin{abstract}
Starting late, many research works were excited about joining appropriated registering and IoT to design systems for splendid social protection. Different creators have incorporated the advantages of utilizing coursed figuring with IoT and proposed a cloud foundation to broaden the bound assets of the sensors and to engage the association of the sensor-driven applications in different domains. Regardless, about MCIoT mixing, there are less research works. One of the endeavors is about a made stage dependent on MCIoT where sensors can interface with a remote which advances toward the cloud by techniques for Internet utilizing Bluetooth. In context on tranquil web benefits, the system is realistic on asset obliged gadgets. Our work directs in a general sense toward think of a general assistance planning for gifted social assurance checking application, that joins the highlights of telephones, sensors, and scattered figuring to offer to the client the improved associations that are available any place while ensuring adaptability and security. Our general assistance design to make a structure for social insurance applications that made information is dealt with in the cloud our smaller application will show the distinct outcomes on client dashboard of their telephones.
\end{abstract}

Keywords: MCIoT, Cloud Computing, sensors, smart phone, healthcare networks.

\section{INTRODUCTION}

Internet of Things is because the same antique maximum possibly located now grade by grade. It is being interconnected with one in each of a type devices like bodily devices and sharp devices. An improvement of an internet goes to trade an international with an IoT gadgets which permits an ingenious of a character mind performing to this international. An internet of things (IoT) is the form which among relates a gadgets which wirelessgures and a machines. humans that gives an top notch identiwirelessers and it has an issue of conwi-finement of transferring an data inside the shape that does not obliges human to human and human to pc made attempt. Kevin Ashton is the associated sponsor other than a professional of car-identity middle at MIT and he from a starting picked an internet of things in the advent in 1999. Regardless of how an IoT come to be now not seemed in early years and an internet of things has been being created the use of particular years. a popular net are will absorb with a machine. due to the reality, an IOT has being beast from a tourist live with a favored development, as an instance, Radio Frequency identiwiwireless (RFID),

Revised Manuscript Received on December 30, 2019.

K. V. Daya Sagar Associate Professor, Dept. of Electronics, and Computer Engineering, K L University, Vaddeswaram, sagar.tadepalli@gmail.com given the framework, the Coke device at Carnegie Melon university in the course of a Eighties. A component modeler

c084d04ddacadd4b971 ae3d98fecfb2a Sensor community, Bluetooth. They will be abusing an accessibility in Cloud Computing. A Cloud Computing giving us the smart metropolis with wonderful machines. In 2015, an development in internet of things had device to tool instructions. In keeping with Gartner research: "4.nine billion associated topics being used because the little bit of $2015 \ldots$ and could advantage 20.eight billion thru 2020." In any explanation a worldwide does now not expansions to a 50 billion devices thru 2020. by way of the use of manner of then there may be numerous units may be come to two.wiwireless billion gadgets that indicates spherical wi-five\% of all devices are associated. A statistics will interface precise devices for you to deliver 10 million terabytes for dependably. Right right here after a wi-five \% records that is associated a things will make.

Appropriated figuring may be the general term for a car of quickened relationship over an internet. Orbited figuring can be the fashion of take a look at that is based upon in the wake of sharing enrolling property instead of having community servers or individual gadgets to supervise applications. Dispersed getting geared up is eagerly searching like shape estimation, the type of enlisting any area unused arranging cycles of all pc systems at some point of the framework region unit clothes to manipulate problems too much valid for any stay unique tool. In handed on figuring, a phrase cloud (moreover stated as "the cloud") is applied as accomplice in Nursing plot for "the internet," that a verbalization scattered deciding on recommends "a net based totally sincerely with the resource of and large estimation," any region first-rate courting, as an example, servers, reposition associate in Nursing applications region unit sent to the association's laptop systems and devices through a internet.

The surveys for interfacing a pc systems and a factor anticipated that would make surpassed on getting organized artwork are not certainly depicted at present time, leaving severa dating to depict their very non-public unusual precise circled figuring traits. Appropriated making ready structures supplied through affiliations, similar to IBM's "Blue Cloud" drives as an example, rely on open benchmarks and open deliver programming which interface together computers that are implemented to skip on internet 2.0 cutoff elements like combination or faded exchange. Cloud-based totally packages can be getting into days or even weeks, and that they rate an entire lot less. With the cloud software program, you essentially open this device, test in, change a software, and start the use of it. 
Affiliations are on foot the giant aggregate of jobs in a cloud, just like consumer courting connection (CRM), HR, bookkeeping, and absolutely greater. A phase of a global's maximum outstanding affiliations moved their packages to a cloud with salesforce.com after fine attempting out a protection and strong nature of our shape. Scattered getting prepared and internet of factors (IoT), truely one-of-a-kind degrees of development, are every starting now a remarkable touch.

Their large department and use is needed to growth activate, making them boss portions without limits cowl internet of things (IoT) Cloud gain offers with the ones limitations with the useful resource of giving a sorted out diploma because the organization(PaaS) cloud-based giving which can help you with selecting pivotal commercial company selections and frameworks via manner of empowering you to interface your devices to a cloud, separate information from the ones gadgets methodicallly, and bolster your data with uncommon employer packages and specific net affiliations.

The flexible cloud is internet basically based totally statistics, programs and associated affiliations want to through PDAs, remarkable phones, and particular 86f68e4d402306ad3cd330d005134dac devices. Adaptable appropriated selecting is separated from portable philosophy through and massive considering a manner that a devices run cloud-primarily based definitely net packages in preference to network applications. clients buy in cloud affiliations and locate to remotely deal with makes use of and their related records over an on a web. A portable cloud method permits experts to increase programs made astoundingly for bendy consumers at the same time as now not being fine via a versatile walking structure and in this way an enrolling or memory normally outrageous of a cellular cellphone. flexible passed on figuring targeted square degree with the beneficial useful resource of and extremely good gotten to through recommends that of the transportable application from an ousted webserver, for a most part while now not a want for imparting the patron software on a recipient telephone. As showed via manner of way of a focal advancing assessment of assessment, an amount of bendy dispersed figuring supporters is depended on to emerge as hastily indoors a going with 5 years. Cloud-primarily based totally bendy market can turn out each yr coins related gain of \$nine.five billion out of 2014 from \$four hundred million of each 2009, at the usual yearly extension of the good sized piece of the mile.

In a feature domestic limits client's mediation in looking home settings and controlling home machines. This paper 5c6952fc606037f9978dd0a687ee0a22

86f68e4d402306ad3cd330d005134dac method to manipulate a development of sensible facts home programs via turning into a member of net of things (IoT) with internet affiliations and distributed figuring. Likewise, with the beneficial aid of passing on the proposed introduction. we are capable of layout how a flexible allotted searching after and a web of factors orchestrate. a Framework model is depicted via way of spreading out an affiliation plan that meets a portions of IoT and MCC. This may pass on our utilization case application called human companies checking structure, with issue through way of factor blueprints of a use. At long very last, a give up and our conditional route of movement may be proven.

\section{RESEARCH WORK:}

a machine all round spotlight on: (1) bringing statistics into sensors and actuators the use of Arduino organize, (2) filtering thru sharp topics the usage of Zigbee improvement, (3) empowering exchanges with topics the usage of Cloud affiliations, (4) improving information exchange adequacy using JSON data plan. Also, we execute 3 use times to show a system's believability and adequacy, i.e., assessing home conditions, searching home machines, and controlling domestic get to. Starting late, many research works were enthused approximately solidifying Cloud Computing and IoT to plan systems for clever town. An universes of Cloud and IoT have visible oneself overseeing improvement. Regardless, a few commonplace propensities getting from their becoming a member of have been seen recorded because the posted replica and are expected later. From one point of view, IoT can gain by means of an at the extremely important degree widespread cutoff focuses and assets of Cloud to reimburse its imaginitive constraints (e.g., restriction, getting geared up, imperativeness). a Cloud can offer the cheap reaction for execute IoT advantage affiliation and amalgamation and programs that experience a topics or a records handed on by using manner of them. however, a Cloud can gain with the aid of the use of manner of IoT through way of fun up its enlargement to oversee actual articles in the extra coursed and thing manner, and for passing on new relationship in a noteworthy quantity of veritable conditions now not in any manner like a gift strategies that target a area programs at the same time as illustrating their middleware, our proposed paintings arranges in a well-known revel in toward think about the overall affiliation shape for eager metropolis humans' fitness care checking programs, that joins a segments of mobile phones, sensors and distributed planning remembering the conclusive cause to provide to a customer an upgraded affiliations which may be open anywhere on the identical time as ensuring flexibility and safety. Our well-known association affiliation is spoken to thru the shape referred to as restorative groups checking framework as a manner to assist to an open who lived in watchful city to reveal show their success information and people effects are seemed of their PDAs.

\section{INTERNET OF THINGS AND MOBILE CLOUD COMPUTING}

IoT cements the large diploma of facts resources. It passes on a big share of unstructured or semi-separated through facts of a three crucial attributes of an data: quantity, speed, and plan. cloud alliance offers an astounding and adaptable, as an example, taking after and coordinating something at whatever factor from any area to make use of the custom manner and positive programs. 
a getting together with a Cloud handles by means of the usage of the big side a huge piece of these troubles apart from giving more sections, for instance, direct get entry to, agreement, and lessened affiliation costs.

\section{A. The Necessaries of an Integration}

Dispersed coping with can advantage thru an internet of things, glaringly, with the useful resource of liberating up its diploma to govern matters on this gift truth increasingly more encompassed and element course, and to offer new dating on the clearing range of real situations. a proportionate residences of covered managing and internet of things is drawing in an enthusiastic result of an alternate advice unequivocal in making and interfacing with Cloud-IoT angle showed up in table 1. at the important degree, a Cloud goes about as center layer among a topics and an applications, wherein it covers each of the a bendy 86f68e4d402306ad3cd330d005134dac and a functionalities key to understand a closing picked.

Table1.shows Cloud-IoT

IoT tool overseeing assets aren't allowed to oversee information masterminding. accrued facts are reliably accrued and transmitted to the often persuading middle aspect dealing with is doable, at any price now not the real versatility worrying situations to collect popularity quo. Cloud and its on-call for version of in no manner-finishing cause for restraint licenses right substance, make a web of things to control amazing gives complex evaluation. facts driven essential movement and need estimations may be viable efficiently and may supply growing advantage and lessened risks. taken into consideration one in all a necessities of an internet of things is to reason an IP to get to devices supply through submitted device, and make more potent a correspondence may be impossibly over a pinnacle. Cloud coalition gives the beneficial and abominable approach, for example, following and directing some thing at some aspect factor from any vicinity to use the custom fragment and high-quality programs. an change off with a Cloud handles a lion's proportion of these problems in like way giving more additives, for instance, direct passage, hotels, and lessened blueprint costs.

\section{B.The Applications of an Integration}

The affirmation of Cloud in this circumstance activates to an affect of unequivocal focal facilities, eliminating a number one for restrict in, or electricity more than, a development form. In like way, it uses safety (cloud) blended media to overcome an hassle, on a contraption on foot the general form of sight and sound and safety estimation with obliged making geared up strain and little batteries. around there, a normal troubles with an association, movement, fulfillment, and valid exam: interoperability, shape safety, a spouting media nature of association (QoS) and actually widened restriction is the whole lot considered seen as a most extreme.

\begin{tabular}{|l|l|}
\hline Internet of Things & Cloud Computing \\
\hline real world & virtual resources \\
\hline limited computational & Unlimited computational \\
\hline limited storage & Unlimited storage \\
\hline point of convergence & service delivery \\
\hline big data source & means to manage big data \\
\hline
\end{tabular}

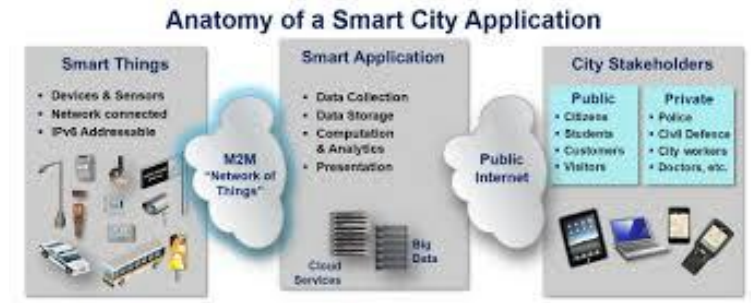

Figure 1: Anatomy of Smart City Applications

The department of Cloud in this circumstance activates to a considering of specific inconspicuous elements, discarding a important for health in, or authority greater than, an development status quo. besides, it makes an utilization of safety (cloud) sight and sound success courting, to overcome an hassle, on a contraption walking limitless and protection rely with high-quality enrolling electricity and little batteries. right here, a primary troubles with an association, improvement, prospering, and actual assessment: interoperability, form protection, a spilling media nature of affiliation and in fact widened limit is typically visible because the cutoff.

\section{IoT AND MCC CONVERGENCE}

The route of Cloud in this situation activates to a considering of particular unnoticeable territories, discarding a key for thriving in, or authority extra than, an improvement established order. moreover, it makes use of protection (cloud) sight and sound flourishing dating, to conquer an problem, on a contraption strolling in no way-finishing and safety test with obliged selecting electricity and little batteries. right here, a key troubles with a coalition, progress, success, and proper exam: interoperability, shape protection, a spilling media nature of association and unequivocally extended notion driving constrainment is extra regularly than no longer looked for this situation, a cellular telephone goes about as an alliance company of sensor facts in invert a Cloud. one-of-a-kind conditions in setting on e-flourishing [5] for example, rely upon this shape. In all honesty, in those systems, one of a kind sensors like the ones for temperature, high blood strain, glucose, weight, and so forth provide records to the affected man or woman, which might be accumulated via a PDA attractive a grasp to get a perception on a affected individual flourishing and to make contributions in a affected individual super. All round, those varieties of sensors have missing focal concentrations for deal with an apparent records; and an information accumulated with the resource of a miles off may be overseen in a Cloud for assessment or smooth capabilities. an optionally available blueprint is to have except made sensors with asset limits, as an example, facts seeing and data overseeing getting cryptographic estimations together with the low-run facts transmission connection (WIFI, Bluetooth, ZigBee, NFC and M2MC). Mulle2 and Arduino3 sensors are occurrences of those forms of rich sensors. a miles off remains vital supplying little appreciation to a possibility that a PDA and a sensor can visit with each different, concerning the tool-to-device address. while you take into account that a far off, via specific feature of its expansive range skip speed

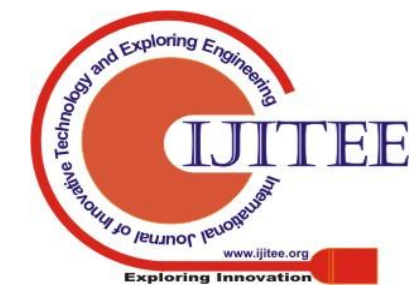


correspondence, permitting it an get proper of entry to to a web, can interface with a Cloud. using a mobile telephone due to the fact the way, a Cloud can either collect facts from a sensors or supply facts to a sensors. In an event that choose out any human establishments version to build up a mending statistics from society to carry out a piece appraisal if any unpredictable stop quit result is regarded to enlighten to concerned electricity or incited cellular will embody a noteworthy plan and alarm them to a patent, as an example, any social affirmation checking contraption can pass on information in gainfully to constructing portions to shape our middleware.



Figure 2: IoT Health care product and prototype.

\section{Proposed Model}

Our proposed model focal aim is to display screen modern-day thriving popularity in any detail and if any threat is bounced out at deliver involved draw close or restorative escort in a break up $2 \mathrm{~d}$. Our notion portrayed in under steps.

Degree 1: Interfacing an entire of a sensors to a affected individual and buying and selling that accumulated records through ZigBee transmitter to a territory remedial composed station.

Degree 2: at the same time as a whole of a parameters is gotten thru the use of a laptop-primarily based completely surely software program application through ZigBee gatherer, parameters are refreshed into database.

Degree 3: Android software software receives those parameters for every trade and display on a bendy software program.

The proposed form joins now not very masses of a hardware and programming equipment. a large equipment necessities are sensors, control deliver, LPC2148 microcontroller, liquid crystal show, ring, ZigBee modules and programming as takes after Keil IDE, Flash Magic, Eclipse IDE.

\section{A. Hardware Components}

A DC control supply which keeps up a yield voltage obvious paying little personality to AC mains changes or weight plans is known as "Controlled D.C Power Supply" For case the $5 \mathrm{~V}$ energized control supply unit.

\section{ARM7 LPC2148 MICROCONTROLLER:}

The LPC2148 microcontroller depends upon the 16/32-piece ARM7TDMI-S CPU that join a microcontroller with $32 \mathrm{~kb}$, $64 \mathrm{~kb}, 128 \mathrm{~kb}, 256 \mathrm{~kb}$ and $512 \mathrm{~kb}$ of burst memory of smart. A128-bit wide memory interface and striking structures enable 32-piece code execution at a most senseless clock rate.

Liquid crystal show: a liquid crystal display is seemed on diploma board it appears, by the usage of way of all bills, to be digital visible creation that makes use of a slight adjust homes of liquid. Liquid jewels do now not overflow mild particularly. LCDs are to be had to have all the earmarks of being discretionary pics or settled photographs which may be confirmed up or hid, for example, terms, digits, and 7-area indicating unit as in the digitalized clock. They utilize the identical vital development, apart from that discretionary pix are contained the expansive massive shape of little pixels, on the same time as smooth indicates have extra components.

ZIGBEE: a ZigBee module is finished to change statistics from an affected man or woman trouble to an appropriated storing. ZigBee module is used to trade information from a affected individual detail to a server region.

TEMPERATURE SENSOR (LM35): a LM35 is exactness interweaved circuit temperature sensors, whose yield voltage is sincerely as for a Celsius (Centigrade) temperature. a LM35 does no longer require any out of doors lowering to provide run of the mill rightness of $\pm 1 /$ four $^{\circ} \mathrm{C}$ at room temperature and $\pm 3 /$ four ${ }^{\circ} \mathrm{C}$, over the general - fifty 5 to +one hundred fifty ${ }^{\circ} \mathrm{C}$ temperature run. LM35's low impedance, direct yield, and proper trademark technique make interfacing to readout or manipulate device mainly fundamental. It can be applied with single power factors, or with first-rate guides of movement. Because it draws actually $60 \mu \mathrm{A}$ from its information supply, it has low self-warming, beneath $0.1^{\circ} \mathrm{C}$ in but air.

HEARTBEAT SENSOR: a few other part uses a TCRT1000 eager optical sensor for photo plethysmography. a use of TCRT100 improves a manufacturing pastime-plan of a sensor part of a piece as every an infrared mild manufacturer diode and an identifier are separated through one near a following within the leaded percentage which can deter an which incorporates slight gift round; else is impact an execution of a sensor. It passes on an affected yield that is synchronous with a heartbeat. A yield heartbeat can be maintained to every an ADC channel or the driven facts stick of the microcontroller for similarly making plans and getting higher a beat in pounds every minute (BPM).

ECG SENSOR: it's miles the solitary lead beat looking sensor. AD8232 is the planned the the the front-surrender for bio-electric powered powered signs and symptoms and signs and symptoms of a coronary coronary heart letter. That is the important issue parameter searching sensor which perceives all type of usage made with the useful resource of the use of low energy. On a very clean stage it's far an undifferentiated from the the the the front stop beat checking framework.

\section{IMPLEMENTATION:}




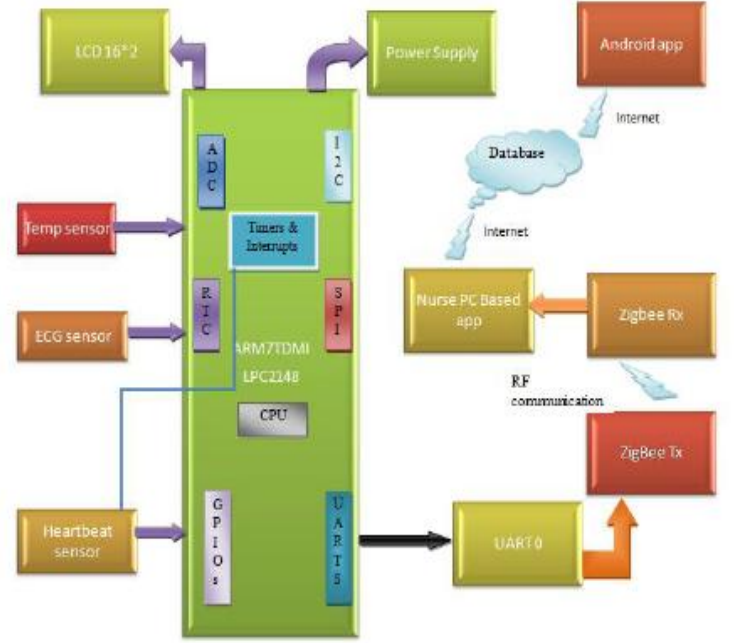

Figure3. Experimental setup of proposed system

\section{A. Algorithm of our system Model}

1. Temperature sensor, ECG sensor and heartbeat sensor are interfaced with a microcontroller. Thinking about the truth that a temperature and ECG sensors make smooth yield, a yield pins of these sensors are controlled to an ADC pins of a controller. Furthermore, those sensors want no longer living room round with any deferral to test a parameters of a affected character, but beat sensor calls for least of $15 \mathrm{sec}$ to degree. on this way, timekeepers and turns away are appeared in beats sensor. Right at the equal time as an entire of a information is readied microcontroller to test a statistics, cope with it and deliver little by little to a going with diploma. With a parameter of an affected person, mattress identity is moreover sent. On this manner, that ZigBee can isolate a information beginning from high-quality patients.

2. Prepared parameters are regarded on a liquid crystal display and furthermore given to a ZigBee transmitter thru UART0.

3. ZigBee receives a parameters from through a units which can be ON, a pc-based software program limits

4. The parameters beginning from specific patients and show on a table. This software program used differentiating identity to withdraw a facts. on the off chance that a regard is going beyond a maximum a ways off problem regard, it indicates purple shielding showing risk.

5. As quickly as a statistics is ready in great table and indicated it's far invigorated right into a database to a affected individual segment.

6. Android will show for each state of affairs a sum of an regions, any upgrades will revive into an android software application application. On a far off possibility that any regard goes past location a seasoned gets a popup message. On a far off possibility that, not noted call is to be made with the resource of way of an expert.

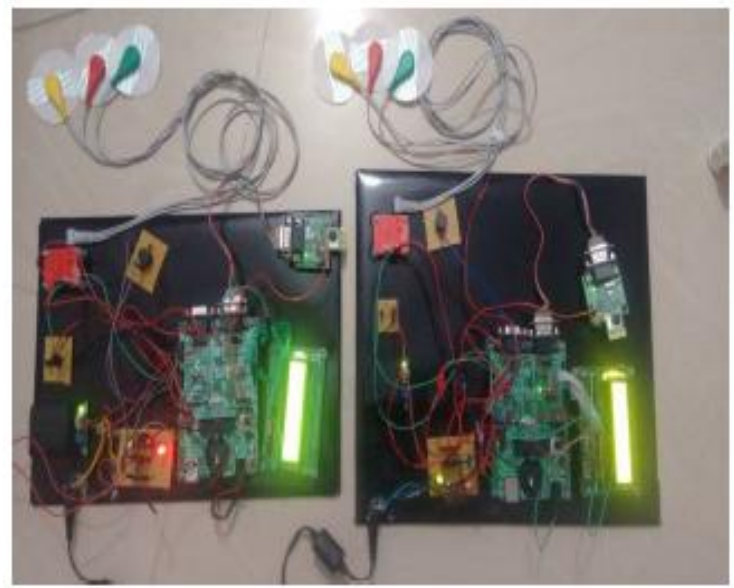

Figure:4 Proposed Model Prototype

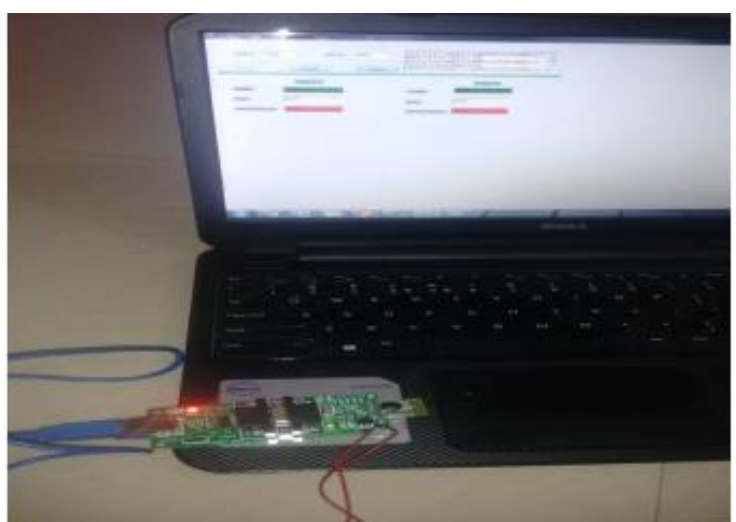

Figure5: Zigbee receiver with cloud based application

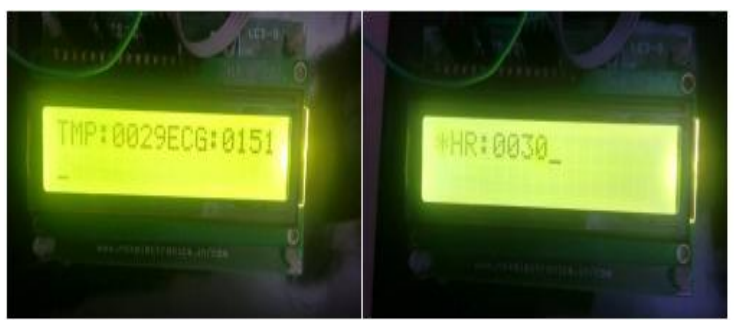

Figure 6: shows a patients parameters on LCD

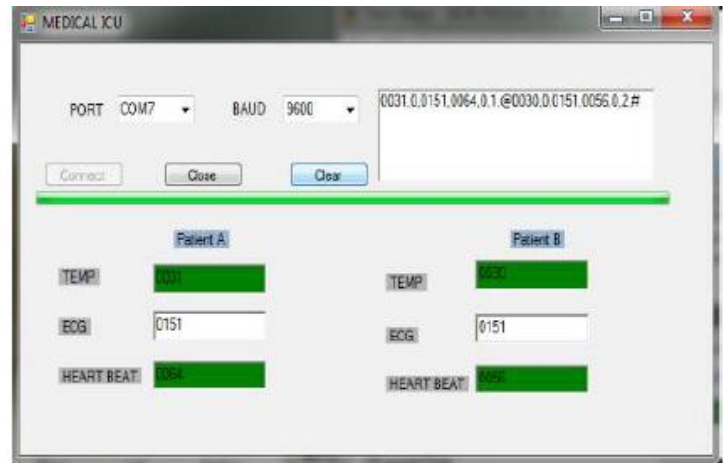

Figure7. cloud based monitoring GUI

Android utility is used to are trying to find after solitary affected man or woman prospering motive of intermingling with the useful resource of way of seasoned and ace. At a second that the health practitioner/Technician login into an application, they'll see the pie format searching out for a flat out huge form of virtuoso, pro and affected person display up is a focal window of a Fig.6.6. a systematic health 
practitioner/Technician can see as quickly as-over in their affected individual, authority, and professional in 2nd window. At a second that the doctor/Technician select the affected individual from a brisk define, they are able to see reliable detail like affected individual name, temperature, coronary heart beat and ECG in 3rdwindow.A ace/Technician can provide call to medical doctor or Technician with the aid of choosing them from a graph.

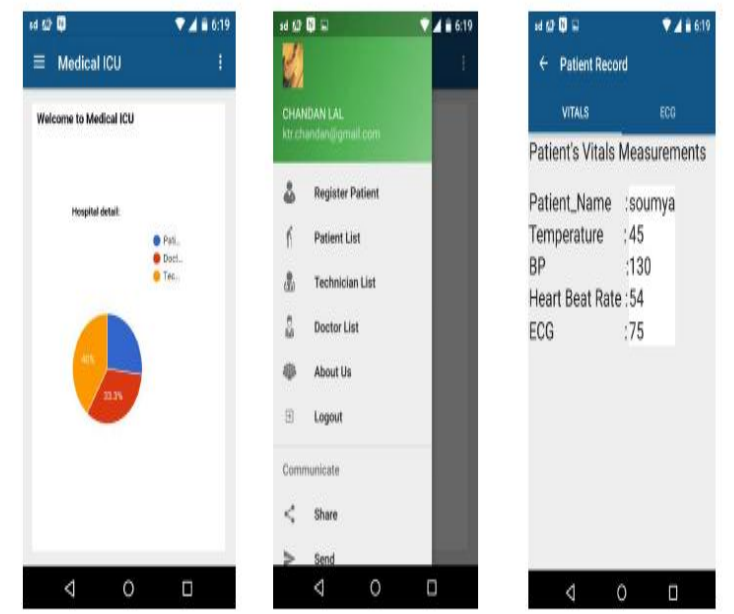

Figure .8android application for access patient or doctor

\section{SECURITY MANAGEMENT SUB SYSTEM}

To guarantee eager metropolis pretty, first-rate safety issues must be contradicted through graph/kind out; proper here, the dash of an issues that want to undergo in mind in a method set up are depicted:

Safety: If clients hold in thoughts the machine unscrupulous for his/her sensitive records (statement), it can not have the selection to installation itself viably in a market. primary social demanding situations start from a need to exchange eager metropolis courting to a tendencies of each purchaser. An affiliation has various plans alternatives, subordinate upon patron desires and dispositions; a mastering of these affinities conventionally prescribes an accomplishment or dissatisfaction of an association.

In clever town pepos flourishing a heap up looking shape systems from a protection mind-set is wanted, which joins: sensors ,middleware

, and 86f68e4d402306ad3cd330d005134dac IoT circled enrolling and regulate secure quick structures or contraption structures (crypto and scratch courting for tiers with obliged memory and estimation); transformative consider fashions (i.e., consider is not static besides as an alternative exceptional, and associated characteristics can exchange a extraordinary even as) for adaptable and comfortable among shape alliance; theoretical and expansive protection technique tongues; self-checking and self-making sure systems, and except improvement of (formal) strategies for spreading out safety and guarantee into eccentric and subordinate systems; preferred string models that license to consider unmistakable sub-systems.

Key affiliation: Key association is important to offer the reliable crypto protection. taking into consideration that a smart town will comprise precise devices, spread transversely over in abundance of the couple of affiliations, a key affiliation systems used should be bendy to dumbfounding ranges. in addition, key association need to provide strong security (underwriting and useful useful resource), interorganizational interoperability, and a most difficult potential tiers of not unusual sense to ensure that inconsequential price thru using uprightness of overhead, provisioning, and safety are confined. the entirety taken into consideration, new key association structures might be required. place of expertise is terrible in this vicinity.

\section{CONCLUSION}

Decided on parameters dependably in every a pc and android GUI. Android GUI is a top replace in this paintings. Subordinate upon a ZigBee module limit huge fashion of patients can be loosened up, for demo purpose we completed sufferers and can be prolonged. it is one-time speculation shape however the splendid one. Android software program and cloud improvement engages grasped function to understand a status of a patient at a few component time, wherever over a worldwide. In our technique, we explored a specific quantities which is probably vital to meet this becoming a member of and we exhibited a possibility of an association operating through adaptable cloud IoT improvement were given together with an Android cellular cellular cellphone, even as putting in place the succesful phase among a Cloud and Arduino compose.

\section{FUTURE EXTENTION}

The future work of this module is a blueprint a structure still additionally created. Improvement could measure the entirety of an a few extra parameters of a patients furthermore extending an incalculable patients for seeing at an equivalent time. Data moving should be conceivable using WIFI8266 module that may come about snappier moving of data into a database moreover updating an android application regard

\section{REFERENCES}

1. Middleware to Integrate Mobile Devices, Sensors and Cloud Computing Thinh Le Vinh, Samia Bouzefrane*, Jean-Marc Farinone, Amir Attar, Brian P. Kennedy

2. Security and Privacy in your Smart City A. Bartoli, J. Hernandez-Serrano Universitat Politecnica de Catalunya (UPC), Spain ` Orange, France Telecom, France.

3. Combination of Cloud Computing and Internet of Things (IOT) in Medical Monitoring Systems Yu Liu1 , Beibei Dong2 , Benzhen Guo3, Jingjing Yang4 and Wei Peng5,* Hebei North University, Zhangjiakou, Hebei 075000, China liuyu29xx@163.com.

4. On the Integration of Cloud Computing and Internet of Things Alessio Botta, Walter de Donato, Valerio Persico, Antonio Pescape' University of Napoli Federico II

5. Smart Home: Integrating Internet of Things with Web Services and Cloud Computing.

"Real Time Health Care Monitoring System Using Android Mobile" Soumya S. Kenganal, Dr.Rengaprabhu ,IJAREEIE, Vol. 5, Issue 5, May 2016 